RAL-95-021

$\mathrm{DTP} / 95 / 14$

February 1995

\title{
Pinning down the Glue in the Proton
}

\author{
A.D. Martin and W.J. Stirling \\ Department of Physics, University of Durham \\ Durham DH1 3LE, England \\ and \\ R.G. Roberts \\ Rutherford Appleton Laboratory, \\ Chilton, Didcot OX11 OQX, England
}

\begin{abstract}
The latest measurements of $F_{2}$ at HERA allow for a combination of gluon and sea quark distributions at small $x$ that is significantly different from those of existing parton sets. We perform a new global fit to deep-inelastic and related data. We find a gluon distribution which is larger for $x \lesssim 0.01$, and smaller for $x \sim 0.1$, and a flatter input sea quark distribution than those obtained in our most recent global analysis. The new fit also gives $\alpha_{s}\left(M_{Z}^{2}\right)=0.114$. We study other experimental information available for the gluon including, in particular, the constraints coming from fixed-target and collider prompt $\gamma$ production data.
\end{abstract}


The advent of the high-energy electron-proton collider, HERA, has enabled the partonic structure of the proton to be investigated in the small $x$ regime, $x \lesssim 10^{-3}$. The first measurements showed that the proton structure function $F_{2}\left(x, Q^{2}\right)$ rose dramatically with decreasing $x$. When these HERA data were included in global analyses of deep-inelastic and related data, the sea quark and gluon distributions were typically found to have the small $x$ behaviour $x S, x g \sim x^{-0.3}$. To be precise, in the most recent MRS analysis [1] the sea quark and gluon distributions are parametrized in the form

$$
\begin{aligned}
x S & =A_{S} x^{-\lambda_{S}}\left(1+\epsilon_{S} \sqrt{x}+\gamma_{S} x\right)(1-x)^{\eta_{S}} \\
x g & =A_{g} x^{-\lambda_{g}}\left(1+\epsilon_{g} \sqrt{x}+\gamma_{g} x\right)(1-x)^{\eta_{g}}
\end{aligned}
$$

with $\epsilon_{g} \equiv 0$ and $\lambda_{S} \equiv \lambda_{g}=\lambda$. At that time there were insufficient experimental constraints on the gluon to justify the introduction of the parameter $\epsilon_{g}$ in $x g$, or to determine the exponent $\lambda_{g}$ independent of that of the sea-quark distribution $S$. The global fit [1] gave $\lambda=0.3 \pm 0.1$ where the value was strongly correlated to $\epsilon_{S}$. An independent global analysis by the CTEQ collaboration [2] gave similar results with a common $\lambda$ of 0.286 . Thus the small- $x$ behaviour of the sea-quark and gluon distributions are closely linked - motivated either by BFKL gluon dynamics with the sea quarks driven by the $g \rightarrow q \bar{q}$ transition, or by GLAP evolution from a low starting $Q^{2}$ scale.

The most recent measurements of $F_{2}\left(x, Q^{2}\right)$ presented by the ZEUS [四] and H1 [5] collaborations have more data points at low $x$ and improved precision compared to those presented earlier. The new measurements are shown in Fig. 1, together with their description by $\operatorname{MRS}(\mathrm{A})$ (11 partons (dash-dot curves). At very small $x$ we see that the $\operatorname{MRS}(\mathrm{A})$ values lie above the new data. The same is true of the new CTEQ3 partons [2] and, to a lesser extent, of the latest set of GRV(94) partons [3] (dotted curves). We therefore repeat the global analysis of Ref. [1] with the new HERA data included. We allow the normalization of the ZEUS [4] and $\mathrm{H} 1$ [0] data sets to vary within their quoted uncertainties ( $\pm 3.5 \%$ and $\pm 4.5 \%$ respectively). The resulting partons, which we denote $\mathrm{A}^{\prime}$, correspond to the following parametrization of the starting distributions at $Q_{0}^{2}=4 \mathrm{GeV}^{2}$

$$
\begin{aligned}
x u_{v} & =A_{u} x^{0.559}(1-0.54 \sqrt{x}+4.65 x)(1-x)^{3.96} \\
x d_{v} & =A_{d} x^{0.335}(1+6.80 \sqrt{x}+1.93 x)(1-x)^{4.46} \\
x S & =0.956 x^{-0.17}(1-2.55 \sqrt{x}+11.2 x)(1-x)^{9.63} \\
x g & =A_{g} x^{-0.17}(1-1.90 \sqrt{x}+4.07 x)(1-x)^{5.33} .
\end{aligned}
$$

The sea $S=2(\bar{u}+\bar{d}+\bar{s}+\bar{c})$ has a flavour structure similar to that of MRS(A), with parameters $A_{\Delta}=0.045, \gamma_{\Delta}=49.6, \eta_{\Delta}=0.3, \delta=0.02$ and $m_{c}^{2}=2.7 \mathrm{GeV}^{2}$ in the notation of Eqs. (7) and (8) of Ref. [1]. The QCD scale parameter is found to be $\Lambda_{\overline{\mathrm{MS}}}\left(n_{f}=4\right)=231 \mathrm{MeV}$, which corresponds to $\alpha_{s}\left(M_{Z}^{2}\right)=0.113$. The parameters $A_{u}$ and $A_{d}$ are determined by the flavour sum rules, giving 2.26 and 0.279 respectively, and $A_{g}=1.94$ is determined by the momentum sum rule.

The $\mathrm{A}$ and $\mathrm{A}^{\prime}$ partons differ only in the small- $x$ region, since the data have changed only in this region. Indeed the main effect of the new HERA data is to change the small- $x$ behaviour

\footnotetext{
${ }^{1}$ The FORTRAN code for the $\mathrm{A}^{\prime}$ and $\mathrm{G}$ sets are available by electronic mail from W.J.Stirling@durham.ac.uk
} 
$\lambda \equiv \lambda_{S}=\lambda_{g}=0.3$ of $\operatorname{MRS}(\mathrm{A})$ to $\lambda=0.17$ of $\operatorname{MRS}\left(\mathrm{A}^{\prime}\right)$. Besides the change in $\lambda$, in the $\mathrm{A}^{\prime}$ analysis we allowed $\epsilon_{g}$ to be a free parameter, which also helped to significantly improve the fit. The dashed curves of Fig. 1 show how the description of the small- $x$ data is improved. However it is evident that the HERA data suggest stronger scaling violations than the new $\mathrm{A}^{\prime}$ fit. Now, for $x \lesssim 0.01$, the slope

$$
\frac{\partial F_{2}\left(x, Q^{2}\right)}{\partial \log Q^{2}} \simeq \frac{\alpha_{s}\left(Q^{2}\right)}{\pi} \sum_{q} e_{q}^{2} \int_{x}^{1} \frac{d y}{y}\left(\frac{x}{y}\right) P_{q g}\left(\frac{x}{y}\right) y g\left(y, Q^{2}\right),
$$

is virtually a direct indicator of the magnitude of the gluon distribution. Indeed both the ZEUS and H1 collaborations [7] have used their respective data to determine the slope, and hence the gluon distribution, and find values for the gluon in excess of $\operatorname{MRS}\left(\mathrm{A}^{\prime}\right)$, more consistent in fact with the steeply rising gluon distributions of, for example, $\operatorname{MRS}\left(\mathrm{D}_{-}^{\prime}\right)[6]$. On the one hand the new $\mathrm{A}^{\prime}$ fit to $F_{2}$ gives a flatter sea (and gluon) and yet on the other hand the slope $\partial F_{2}\left(x, Q^{2}\right) / \partial \log Q^{2}$ seems to prefer a steeper gluon.

The situation is well illustrated by Fig. 2. The upper plot for $\partial F_{2}\left(x, Q^{2}\right) / \partial \log Q^{2}$ is an indicator of the gluon, see Eq. (7), whereas the lower plot is dominated by the sea quark distribution. The new HERA data show that the dashed $\mathrm{A}^{\prime}$ curve, obtained assuming $\lambda_{g}=\lambda_{S}$, is too flat (steep) in the upper (lower) plot. We conclude that the small- $x$ data are now sufficiently precise that their description requires partons with $\lambda_{g} \neq \lambda_{S}$. We therefore repeat the global analysis with both $\lambda_{g}$ and $\lambda_{S}$ free and find at $Q_{0}^{2}=4 \mathrm{GeV}^{2}$

$$
\begin{aligned}
x u_{v} & =A_{u} x^{0.593}(1-0.76 \sqrt{x}+4.20 x)(1-x)^{3.96} \\
x d_{v} & =A_{d} x^{0.335}(1+8.63 \sqrt{x}+0.32 x)(1-x)^{4.41} \\
x S & =1.74 x^{-0.067}(1-3.45 \sqrt{x}+10.3 x)(1-x)^{10.1} \\
x g & =A_{g} x^{-0.301}(1-4.14 \sqrt{x}+10.1 x)(1-x)^{6.06}
\end{aligned}
$$

with $A_{g}=1.51 ; A_{\Delta}=0.043$ and $\gamma_{\Delta}=64.9$, the remaining parameters being unchanged from $\mathrm{A}^{\prime}$. The QCD scale parameter increases to

$$
\Lambda_{\overline{\mathrm{MS}}}\left(n_{f}=4\right)=255 \mathrm{MeV},
$$

which corresponds to $\alpha_{s}\left(M_{Z}^{2}\right)=0.114$. The resulting set of partons ${ }^{1}$ is denoted by $\operatorname{MRS}(\mathrm{G})$, and gives rise to the continuous curves in Figs. 1 and 2. We see that the steeper gluon $\left(\lambda_{g}=0.30\right)$ and the flatter sea $\left(\lambda_{S}=0.07\right)$ significantly improve the description of the small- $x$ HERA data. Due to the negative $\epsilon_{g} \sqrt{x}$ term in $x g$, the effective value of $\lambda_{g}$ is larger than the value shown. To be precise, if we approximate Eq. (11) by $x g=A x^{-\bar{\lambda}}$ over the interval $10^{-4}<x<5 \times 10^{-3}$ then we find $\bar{\lambda}=0.355$. Note that the data shown in Fig. 2(b) are the result of extrapolating measured data from higher values of $Q^{2}$. Even if future low $Q^{2}$ measurements continue to show a rise for $F_{2}$ at small $x$ the slopes extracted from Fig. 2(a) would still allow a steep gluon solution.

The new HERA measurements of $F_{2}$ provide for the first time a reliable estimate of the gluon at small $x$. We can immediately see the trends. If it is indeed true that the HERA data prefer an enhancement of the $\mathrm{A}^{\prime}$ gluon for $x \sim 10^{-3}$ [7] then there are implications for other gluon-dominated reactions, in particular for prompt $\gamma$ production. The fixed-target prompt $\gamma$ 
data [8, 9] pin the gluon at $x \sim 0.4$. This information, together with the momentum sum rule, implies (i) a decrease of the gluon at medium $x(\sim 0.1)$ and (ii) a steeper slope of the gluon from $x \sim 10^{-1}$ to $x \sim 10^{-3}$. As a consequence we find (see below) that the gluon obtained from our new global analysis $\mathrm{G}$ does indeed change the description of the $p \bar{p}$ collider data on prompt photon production at small $x_{T}=2 p_{T}^{\gamma} / \sqrt{s}$.

Another important consequence of the reduction of the gluon in the interval $x \sim 0.1-0.2$ concerns the prediction for the value of $\alpha_{s}$. At these $x$ values the gluon still gives a significant (positive) contribution to $\partial F_{2} / \partial \log Q^{2}$. Since precise fixed-target measurements of $F_{2}$ exist in this $x$ region (see for example Figs. 6 and 8 of Ref. [1]), the reduction in the gluon must be compensated by an increase in the value of $\alpha_{s}$. This brings the deep-inelastic determination of $\alpha_{s}=0.114$ more in line with the world average value $\alpha_{s}=0.117$ [10].

Figure 3 compares the $\mathrm{A}, \mathrm{A}^{\prime}$ and $\mathrm{G}$ partons at $Q^{2}=20 \mathrm{GeV}^{2}$. We see that the quark distributions are essentially identical for $x>0.05$. Indeed the $\mathrm{A}^{\prime}$ and $\mathrm{G}$ description of the fixed-target deep-inelastic scattering data, the $W^{ \pm}$rapidity asymmetry and the asymmetry of Drell-Yan production in $p p$ and $p n$ collisions is as good as that obtained by MRS(A), see Ref. [1]. However from Fig. 3 we see a sizeable difference in the $A / A^{\prime}$ and $G$ small- $x$ gluon distributions. Fig. 4 highlights this difference, as well as indicating the $x$ range of the various experimental constraints on the gluon. Comparing the $\mathrm{G}$ and $\mathrm{A}^{\prime}$ gluons, we see (i) that the new HERA measurements of $\partial F_{2} / \partial \log Q^{2}$ (Fig. 2) lead to an enhancement of the gluon for $x \lesssim 0.01$, (ii) that the fixed-target prompt photon data require the gluon to be unchanged for $0.35 \lesssim x \lesssim 0.55$, see Fig. 5 , and (iii) that as a consequence the G gluon is reduced in the intermediate interval $0.02 \lesssim x \lesssim 0.2$. It is also interesting to compare the fraction of the gluon's momentum in various intervals of $x$ for $Q^{2}=20 \mathrm{GeV}^{2}$ for the two sets of partons:

\begin{tabular}{|c|c|c|c|c|}
\hline set & $0<x<10^{-3}$ & $10^{-3}<x<10^{-2}$ & $10^{-2}<x<10^{-1}$ & $10^{-1}<x<1$ \\
\hline $\operatorname{MRS}\left(\mathrm{A}^{\prime}\right)$ & $3.5 \%$ & $13.4 \%$ & $46.1 \%$ & $37.0 \%$ \\
$\operatorname{MRS}(\mathrm{G})$ & $5.9 \%$ & $16.0 \%$ & $42.3 \%$ & $35.7 \%$ \\
\hline
\end{tabular}

Prompt photon production serves as a strong constraint on the gluon since it contributes at leading order. As mentioned above, the previous MRS analyses have used the WA70 fixed-target data [8] to pin down the gluon for $x \sim 0.4$. The procedure adopted was to perform a nextto-leading order fit with the renormalization and factorization scales optimized as described in Ref. 11]. There now exists quite a range of prompt photon data which cover the interval $0.01 \lesssim x \lesssim 0.6$. Following Ref. [12], we display these data in Fig. 5(a), together with their next-to-leading order description by the new $\mathrm{G}$ and $\mathrm{A}^{\prime}$ parton sets with the renormalization and factorization scales set to $\mu=0.5 p_{T}^{\gamma}$. In our plot we show the more recent ISR data from the R807 collaboration [14], rather than the earlier R806 measurements. We note that these improved ISR data have a $p_{T}^{\gamma}$ distribution whose shape is more in accord with QCD than those of R806. From Fig. 5 we see that, in general, the next-to-leading order QCD description of the prompt photon data is satisfactory, except that the $p_{T}^{\gamma}$ distributions observed by the CDF [13] and UA2 15 collaborations are steeper than is predicted. Scale ambiguities could remedy a discrepancy in normalization but cannot significantly change the shape of a distribution. On the other hand the description of prompt photon production at high energies and small $p_{T}^{\gamma}$ is complicated by the presence of sizeable photon bremsstrahlung contributions. A detailed study 
[16 of these photonic fragmentation effects (based on the full next-to-leading order calculations of Refs. [17]) has been made, and as a result the QCD predictions are improved. However the description of the CDF and UA2 data shown in Fig. 5 already includes these next-to-leading order effects of photon fragmentation and, although the shape is improved, it is still not satisfactory. It was hoped that the steeper $\operatorname{MRS}(\mathrm{G})$ gluon would have further improved the description of these data. We see from comparing the two plots of Fig. 5 that the improvement is, in fact, marginal. The most probable explanation [12 of the residual discrepancy in the shape of the CDF and UA2 distributions at small $x_{T}$ is the presence of intrinsic $k_{T}$ arising from nonperturbative or 'soft' perturbative effects. Since no soft resummation calculations are available, we have modelled these effects by a two-dimensional transverse momentum convolution of the next-to-leading order QCD prediction for the $p_{T}^{\gamma}$ distribution with a Gaussian intrinsic $k_{T}$ form, $\exp \left(-k_{T}^{2} / k_{0}^{2}\right)$, and varied the parameter $k_{0}^{2}$ to give the best fit to each set of prompt photon data. We find that an excellent description of the shape of the CDF and UA2 data sets can then be obtained if $\left\langle k_{T}\right\rangle=\sqrt{\pi} k_{0} / 2$ is taken to be $2.4 \mathrm{GeV}$ in both cases.

What other scattering processes could provide information on the gluon distribution at small $x$ ? In the production of $b \bar{b}$ pairs in high-energy hadron-hadron collisions the gluon enters (quadratically) at leading order via the subprocess $g g \rightarrow b \bar{b}$. The prediction for the cross section is however very sensitive to the choice of $m_{b}$ and the QCD scale, even at next-to-leading order, and reasonable variations of these parameters can change the normalization by a factor of 2 [18. Furthermore it is difficult experimentally to accurately reconstruct the $b$-quark cross section from measurements of its decay products $(B, J / \psi$, leptons, ...). We have checked that for $p \bar{p}$ collisions at $\sqrt{s}=1.8 \mathrm{TeV}$ the differences in the $\mathrm{A}^{\prime}$ and $\mathrm{G}$ gluons at small $x$ only reveal themselves at $p_{T}(b) \lesssim 10 \mathrm{GeV} / c$ where, unfortunately, there are essentially no CDF or D0 data. At higher $p_{T}(b)$ the shape of the $p_{T}$ distribution measured by CDF [18] is in reasonable agreement with the theoretical predictions, although the normalization is slightly larger than predicted with 'canonical' scale and mass values.

Dijet production in $p \bar{p}$ collisions can also, in principle, probe the small $x$ gluon [20, 21, 22]. For example, if two jets are produced with equal transverse momentum $p_{T}$ and pseudorapidity $\eta \gg 1$ then $x_{1} \sim 1, x_{2} \sim 2 p_{T} / \sqrt{s} \exp (-\eta) \ll 1$. A detailed calculation [19] shows that at $\sqrt{s}=1.8 \mathrm{TeV}$ the gluon can be probed in this way in the range $0.005 \lesssim x_{g} \lesssim 0.05$, as indicated in Fig. 4. However, at present the systematic errors are too large to allow any definite conclusion to be drawn.

In a large region of phase space, inelastic $J / \psi$ production at HERA should be dominated by the 'colour singlet' process $\gamma g \rightarrow J / \psi g$ [23]. Here the gluon is probed at $x \approx c M_{\psi}^{2} / s_{\gamma p} \ll 1$, where the constant $c$ depends on the cuts used to define the inelastic events [24]. The theoretical calculation has recently been extended to next-to-leading order [25]. Forthcoming data from HERA, with for example $50 \lesssim \sqrt{s_{\gamma p}} \lesssim 150 \mathrm{GeV}$ and taking $c=3.4$ [24, will allow us to probe the interesting small $x \sim 10^{-3}-10^{-2}$ region, although it remains to be seen whether the precision will be high enough to discriminate between gluons like those of $\mathrm{A}^{\prime}$ and $\mathrm{G}$.

Note that although there is a large overlap in the $x$ region probed by $p \bar{p}$ dijet and HERA $J / \psi$ production, the $Q^{2}$ values are very different, being respectively $Q^{2} \sim 10^{3}-10^{4} \mathrm{GeV}^{2}$ and $Q^{2} \sim 10 \mathrm{GeV}^{2}$. Since the gluon distributions tend to become more similar as $Q^{2}$ increases, the

\footnotetext{
${ }^{2}$ We thank Werner Vogelsang for providing these predictions

${ }^{3}$ We thank Slawek Tkaczyk for performing these calculations
} 
$J / \psi$ cross section at lower $Q^{2}$ provides greater discrimination. For example, the ratio of the $\mathrm{G}$ and $\mathrm{A}^{\prime}$ gluons at $x=10^{-3}$ is 1.71 at $Q^{2}=5 \mathrm{GeV}^{2}$ and only 1.11 at $Q^{2}=5 \times 10^{3} \mathrm{GeV}^{2}$. The difference at the former $Q^{2}$ value is indicative of the difference in the inelastic $J / \psi$ cross section predictions at high $\sqrt{s_{\gamma p}}$ at HERA.

In conclusion, we have seen that standard sets of next-to-leading order parton distributions like MRS(A), CTEQ3 and GRV(94) give a good overall description of the new HERA structure function data. However at the very smallest $x$ values there is evidence that a new effect is becoming apparent, namely that the small- $x$ behaviour of the gluon and sea-quark distributions are not linked at $Q^{2}=5 \mathrm{GeV}^{2}$. If, as in set $\mathrm{MRS}\left(\mathrm{A}^{\prime}\right)$, we force the distributions to be the same, then the slope $\partial F_{2}\left(x, Q^{2}\right) / \partial \log Q^{2}$ at small $x$ is underestimated. Our new $\operatorname{MRS}(\mathrm{G})$ set accommodates this by allowing the small- $x$ quarks and gluons to have a different shape in $x$. In the notation of Eqs. (1) and (2) we find that $\lambda_{g}=0.30$ and $\lambda_{S}=0.07$, though the precise values are correlated to the parameters $\epsilon_{g}$ and $\epsilon_{S}$. The situation is summarized in Fig. 6, where we show the theoretical predictions and experimental measurements of the structure function $F_{2}$ at a 'typical' small- $x$ value, $x=0.0004$. The $\mathrm{A}^{\prime}$ and $\mathrm{G}$ curves are labelled with their gluon and singlet quark effective $\lambda$ values at this $x$ and the starting value $Q^{2}=4 \mathrm{GeV}^{2}$.

The resulting $\operatorname{MRS}(\mathrm{G})$ 'singular' gluon and 'flat' sea quark distributions do not have a ready explanation in terms of either perturbative or non-perturbative QCD. On the one hand GLAP evolution from a low scale, such as performed by GRV [3] (see also [26]), develops both a steep gluon and a steep sea quark distribution at small $x$. The former is evident in the data, the latter is not. Again Fig. 6 summarizes the situation. The GRV(94) curve has a similar slope to $\operatorname{MRS}(\mathrm{G})$ (both gluons are steep) but overestimates the data (the GRV quarks are steeper than the $\mathrm{G}$ quarks). On the other hand it might be argued that the leading $\log (1 / x)$ resummation, encapsulated in the BFKL equation, is more appropriate at small $x$. A singular (unintegrated) gluon is obtained, as required by the data, but again the steepness is fed, in this case via the $k_{T}$-factorization theorem, directly into the sea quark distribution and hence into $F_{2}$. Of course the application of next-to-leading order, leading-twist QCD in the HERA small- $x$ regime may be too naive. There are some indications that higher-order perturbative corrections may be non-negligible and that these may affect the structure function and its $Q^{2}$ evolution in different ways [27]. It is not impossible, for example, that the $x \rightarrow 0$ behaviour of the quark and gluon distributions are more similar when higher-order corrections are fully taken into account. Unfortunately the theoretical technology necessary to investigate this with any precision is not yet available. If, on the other hand, the form of the starting distributions at $Q_{0}^{2}$ were primarily of non-perturbative origin, the dominance of the forward quark-proton and gluon-proton scattering amplitudes by pomeron exchange would again have suggested $\lambda_{S} \sim \lambda_{g}$. In any case, more precise structure function measurements at small $x$ and moderate $Q^{2}$ will be very useful in shedding further light on this issue.

\section{Acknowledgements}

We thank Werner Vogelsang for information concerning prompt photon production and Andreas Vogt for providing the code of the latest set of GRV partons. 


\section{References}

[1] A.D. Martin, R.G. Roberts and W.J. Stirling, Phys. Rev. D50 (1994) 6734

[2] CTEQ collaboration: H.L. Lai et al., Michigan State University preprint, MSU-HEP-41024 (1994)

[3] M. Glück, E. Reya and A. Vogt, Dortmund preprint DO-TH 94/24 (1994)

[4] ZEUS collaboration: M. Derrick et al., DESY preprint 94-143 (1994)

[5] H1 collaboration: T. Ahmed et al., DESY preprint 95-006 (1995)

[6] A.D. Martin, R.G. Roberts and W.J. Stirling, Phys. Lett. B306 (1993) 145

[7] ZEUS collaboration: M. Derrick et al., DESY preprint 94-192 (1994)

H1 collaboration: I. Abt et al., Phys. Lett. B321 (1994) 161; G. Rädel, Proc. 27th Int. Conf. on High Energy Physics, Glasgow, July 1994, eds. P.J. Bussey and I.G. Knowles

[8] WA70 collaboration: M. Bonesini et al., Z. Phys. C38 (1988) 371

[9] UA6 collaboration: G. Sozzi et al., Phys. Lett. B317 (1993) 243

[10] B.R. Webber, Proc. 27th Int. Conf. on High Energy Physics, Glasgow, July 1994, eds. P.J. Bussey and I.G. Knowles

[11] P. Aurenche et al., Nucl. Phys. B286 (1987) 553; Phys. Rev. D39 (1989) 3275

[12] J. Huston, E. Kovacs, S. Kuhlmann, H.L. Lai, J.F. Owens and W.-K. Tung, Michigan State University preprint, MSU-HEP-41027 (1995)

[13] CDF collaboration: F. Abe et al., Phys. Rev. Lett. 73 (1994) 2662

[14] AFS collaboration (R807): T. Akesson et al., Sov. J. Nucl. Phys. 51 (1990) 836

[15] UA2 collaboration: J. Alitti et al., Phys. Lett. B299 (1993) 174

[16] M. Glück, L.E. Gordon, E. Reya and W. Vogelsang, Phys. Rev. Lett. 73 (1994) 388

[17] P. Aurenche, P. Chiappetta, M. Fontannaz, J.Ph. Guillet and E. Pilon, Nucl. Phys. B399 (1993) 34

L.E. Gordon and W. Vogelsang, Phys. Rev. D48 (1993) 3136; Phys. Rev. D50 (1994) 6753

[18] CDF collaboration: F. Abe et al., Phys. Rev. Lett. 71 (1993) 500, 71 (1993) 2396

[19] E.W.N. Glover, private communication

[20] CDF collaboration: F. Abe et al., FERMILAB-CONF-93-203-E, Proc. 16th International Symposium on Lepton and Photon Interactions, Ithaca, NY, 10-15 Aug 1993

[21] A.D. Martin, R.G. Roberts and W.J. Stirling, Phys. Lett. B318 (1993) 184 
[22] W.T. Giele, E.W.N. Glover and D.A. Kosower, Phys. Rev. Lett. 73 (1994) 2019, Phys. Lett. B339 (1994) 181, University of Durham preprint DTP-94-102 (1994)

[23] E.L. Berger and D. Jones, Phys. Rev. D23 (1981) 1521

R. Baier and R. Rückl, Phys. Lett. B102 (1981) 364

[24] A.D. Martin, C.-K. Ng and W.J. Stirling, Phys. Lett. B191 (1987) 200

[25] M. Krämer, M. Zunft, J. Steegborn and P.M. Zerwas, DESY preprint DESY-94-207 (1994)

[26] R.D. Ball and S. Forte, Phys. Lett. B336 (1994) 77; preprint CERN-TH-7422-94 (1994), CERN-TH-95-1 (1995)

[27] R.K. Ellis, E. Levin and Z. Kunszt, Nucl. Phys. B420 (1994) 517

R.K. Ellis, F. Hautmann and B.R. Webber, Cavendish Laboratory preprint CAVENDISHHEP-94-18 (1995) 


\section{Figure Captions}

[1] The (a) ZEUS [国] and (b) H1 [5] measurements of $F_{2}\left(x, Q^{2}\right)$ in the small $x$ regime compared with the predictions of the $\operatorname{MRS}(\mathrm{A})$ partons of Ref. [1] (dash-dot curve, shown only for $\left.x \leq 5.6 \times 10^{-4}\right)$, the $\operatorname{MRS}\left(\mathrm{A}^{\prime}\right)$ and $\operatorname{MRS}(\mathrm{G})$ partons of this work (dashed and solid curves respectively), and the GRV(94) partons of Ref. [3] (dotted curve).

[2] (a) The slopes, $b(x)$, determined from linear fits, $F_{2}=a+b \log Q^{2}$, to the HERA data and (b) the values of $F_{2}\left(x, Q^{2}=5 \mathrm{GeV}^{2}\right)$ obtained by extrapolating the linear fits back to $Q^{2}=5 \mathrm{GeV}^{2}$. Also shown are the predictions of the $\operatorname{MRS}\left(\mathrm{A}^{\prime}\right)$ (dashed curve) and $\mathrm{MRS}(\mathrm{G})$ (solid curve) partons of this work, together with the description given by the recent GRV(94) partons of Ref. [3] (dotted curve). The slopes are calculated at $Q^{2}=20 \mathrm{GeV}^{2}$.

[3] $\operatorname{MRS}(\mathrm{G})$ partons compared to $\operatorname{MRS}\left(\mathrm{A}^{\prime}\right)$ and also to the earlier $\operatorname{MRS}(\mathrm{A})$ [1] partons, at $Q^{2}=20 \mathrm{GeV}^{2}$. For clarity, the gluons are shown for $x<0.005$ only.

[4] The $\operatorname{MRS}\left(\mathrm{A}^{\prime}\right)$ and $\operatorname{MRS}(\mathrm{G})$ gluon distributions at $Q^{2}=20 \mathrm{GeV}^{2}$. Also shown are the $x$ intervals in which the gluon is constrained by the various sets of data.

[5] The description of WA70 [8], UA6 [9], R807 [14, UA2 [15] and CDF [13] prompt photon data by the $\operatorname{MRS}\left(\mathrm{A}^{\prime}\right)$ (lower figure) and $\operatorname{MRS}(\mathrm{G})$ (upper figure) partons. The predictions for the $p \bar{p}$ collider cross sections are calculated using the next-to-leading order photonic fragmentation program of Ref. [16], and for the other cross sections using the next-toleading order program of Aurenche et al. [17].

[6] The description of the ZEUS [4] and H1 [5] measurements of $F_{2}\left(x, Q^{2}\right)$ near $x=0.0004$ compared with the $\operatorname{MRS}\left(\mathrm{A}^{\prime}\right)$ and $\mathrm{MRS}(\mathrm{G})$ partons of this work (dashed and solid curves respectively), and the GRV(94) partons of Ref. [3] (dotted curve). The effective $\lambda$ values for the gluon and singlet quark distributions at this value of $x$ and $Q^{2}=4 \mathrm{GeV}^{2}$ are indicated. 
Zeus (x1.02) comparison at small $x$
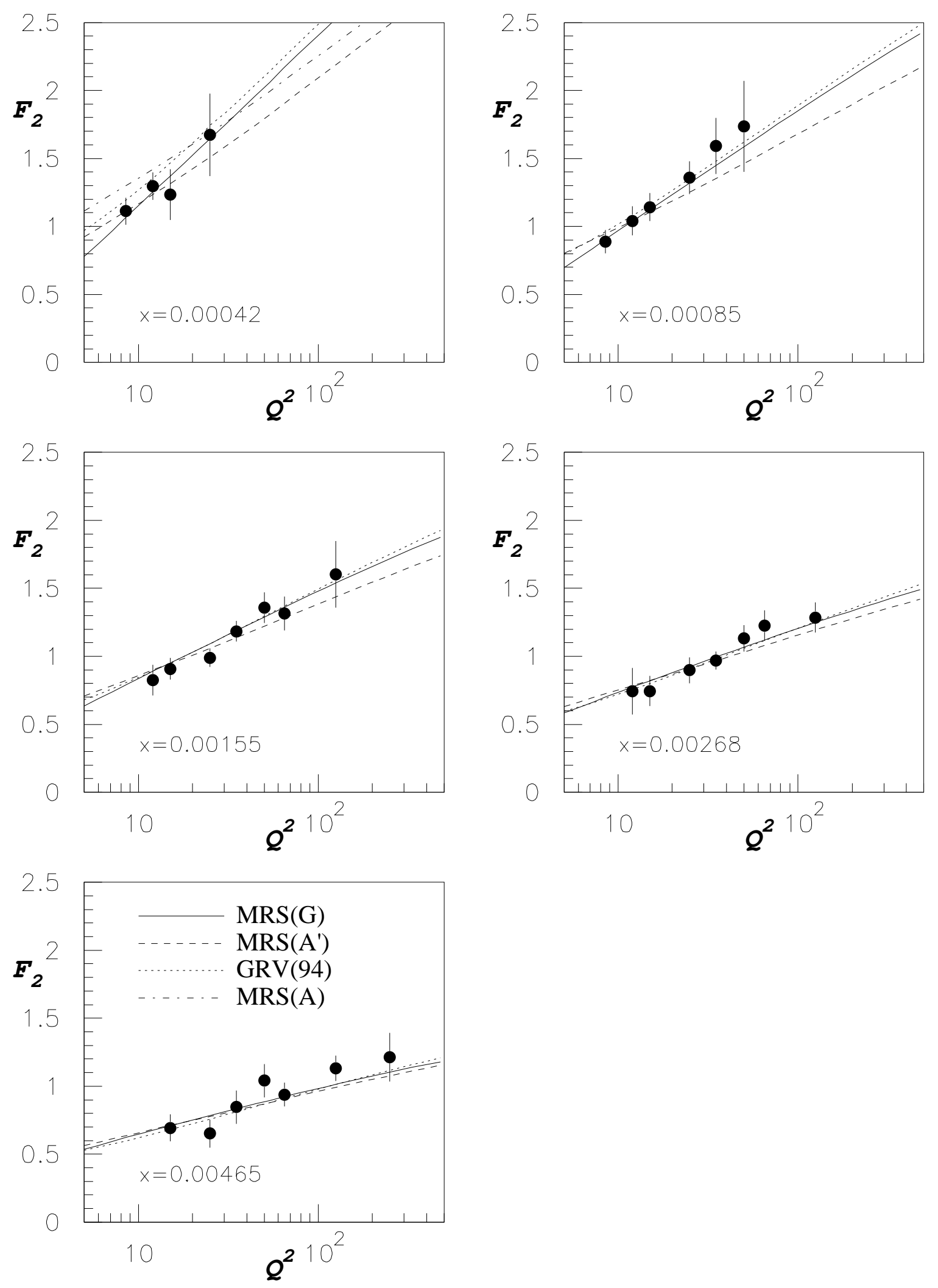

Fig. 1 (a) 
H1 (x0.96) comparison at small $x$
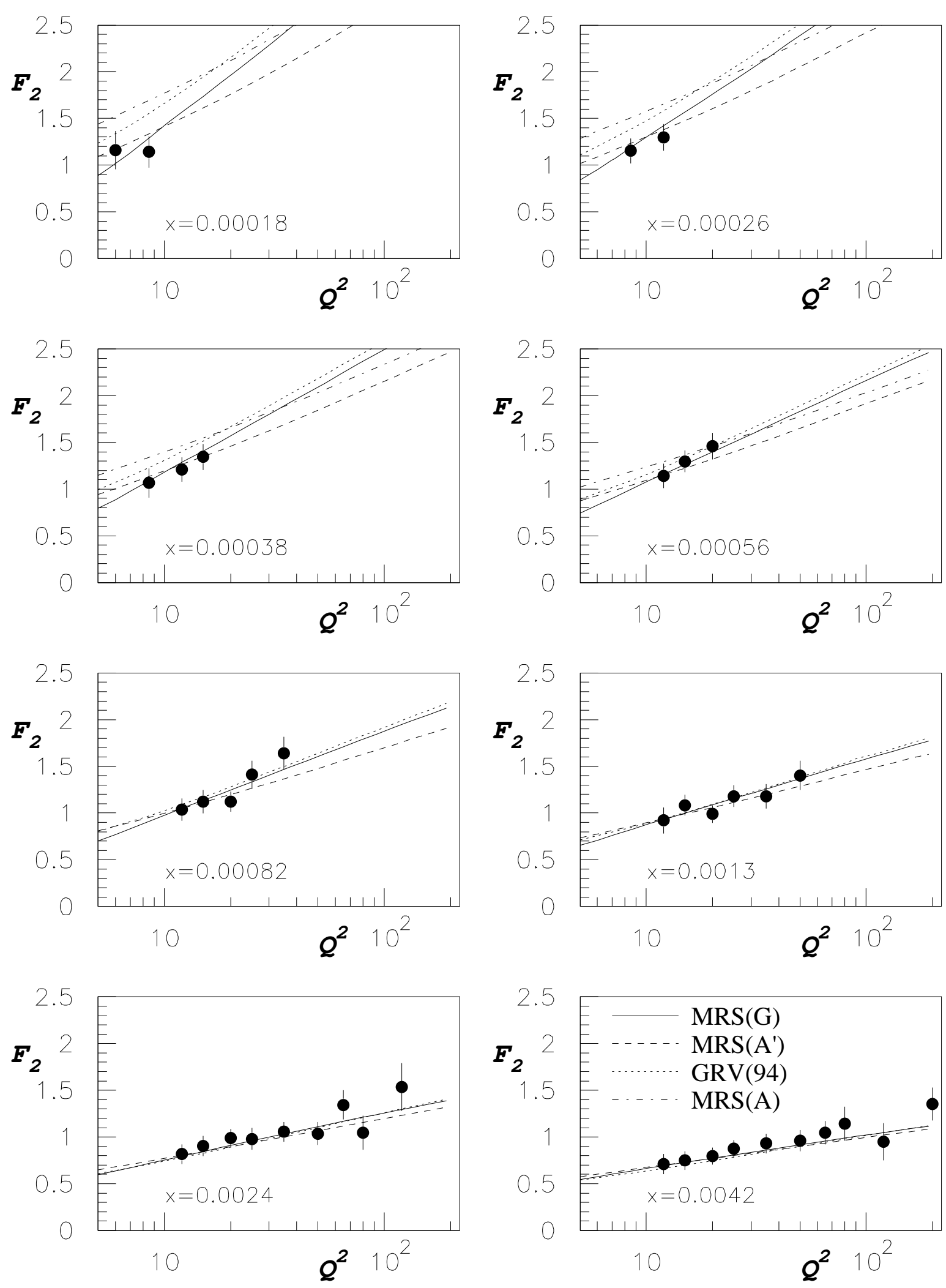

Fig. 1 (b) 
$Q^{2}$ derivative of $F_{2}$
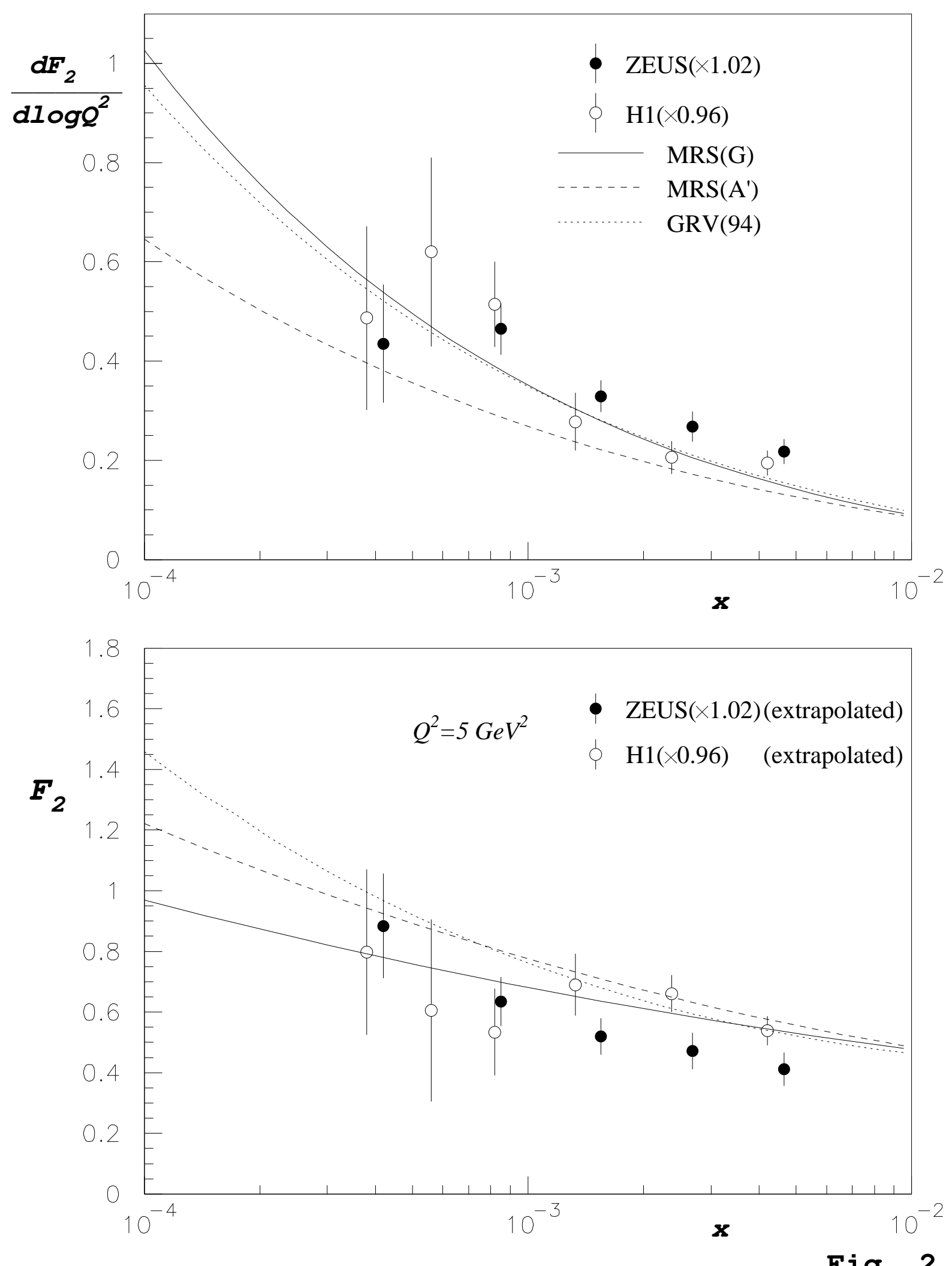

Fig. 2 


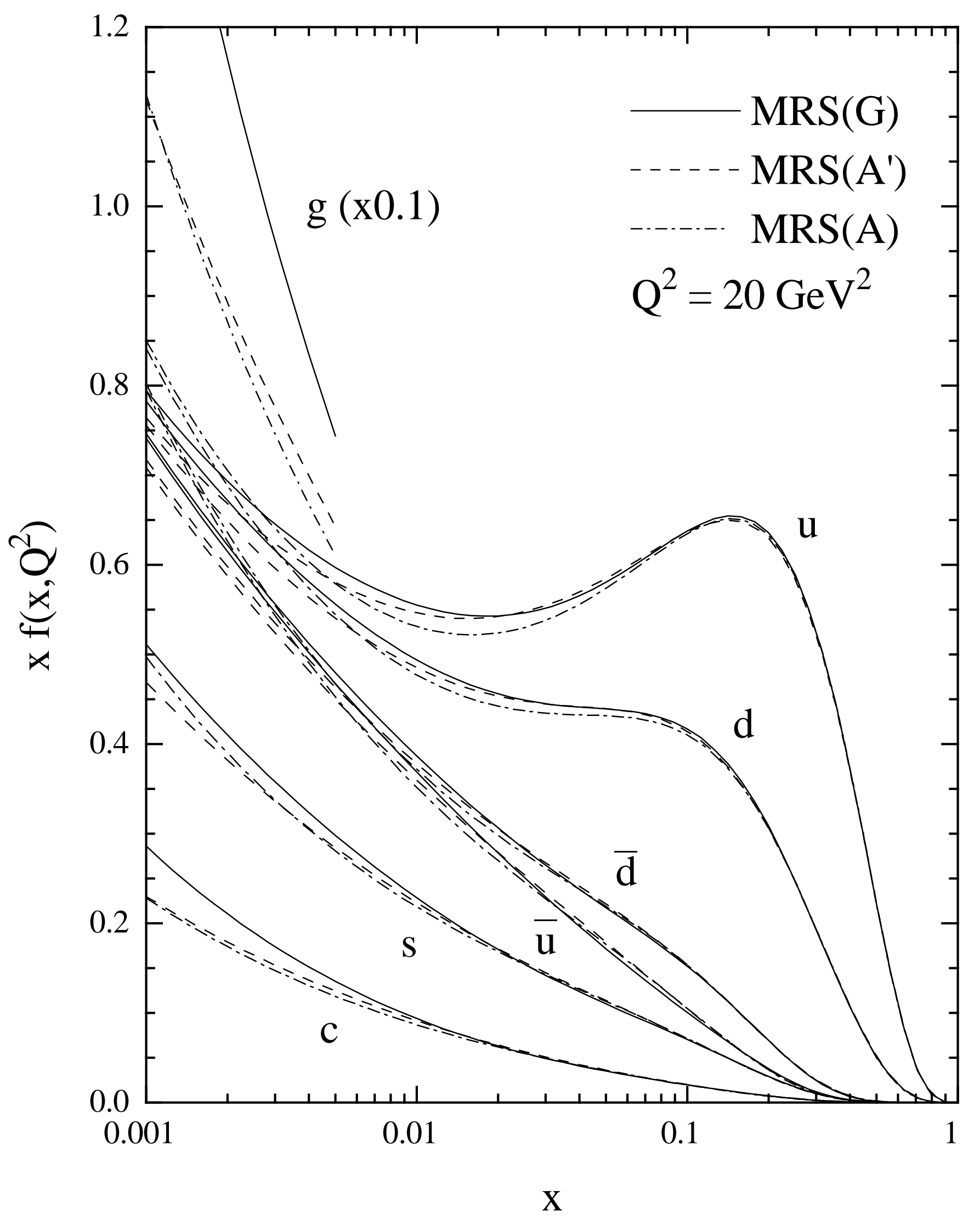

Fig. 3 


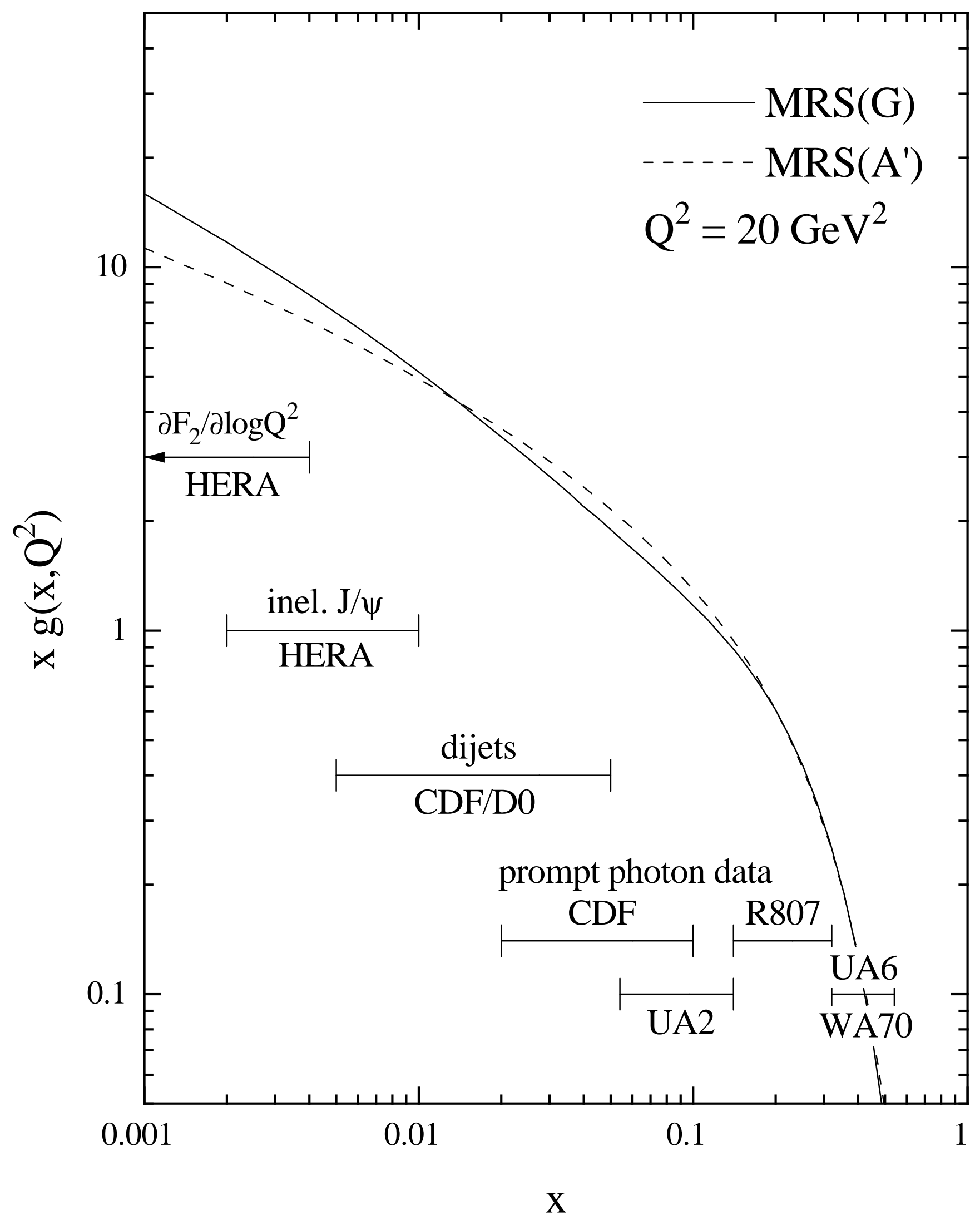

Fig. 4 
Prompt Photon Production
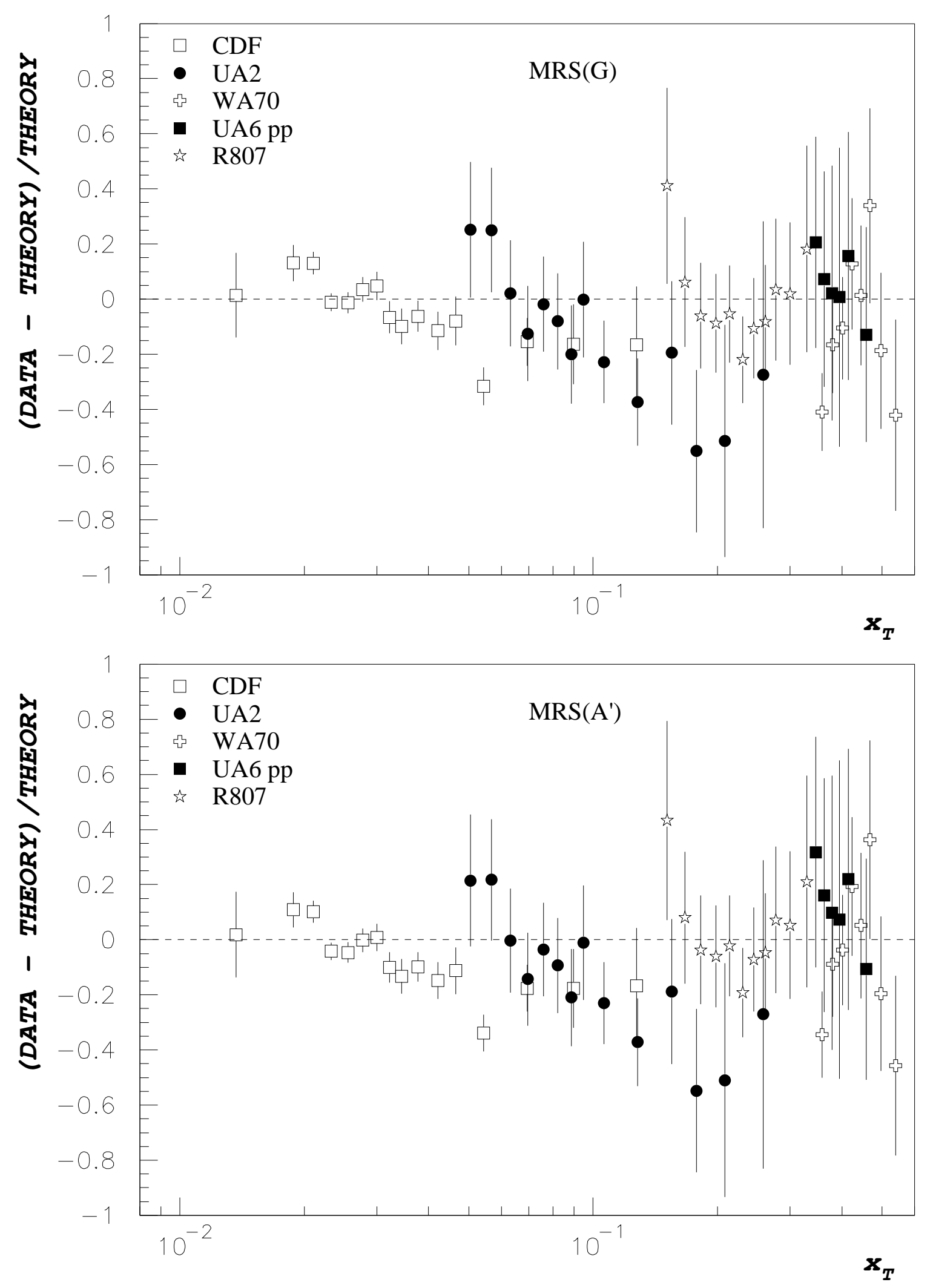

Fig. 5 


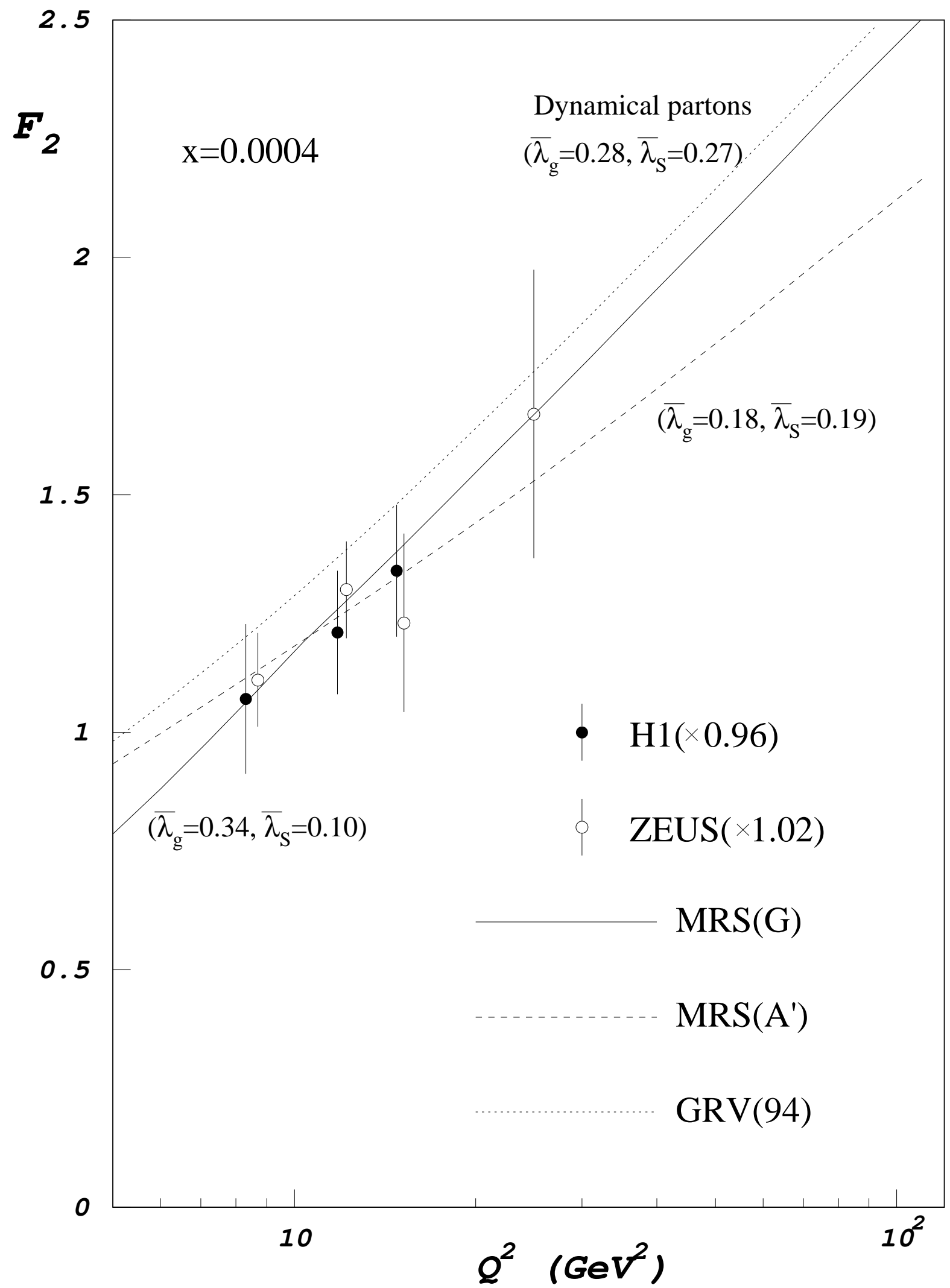

Fig. 6 